\title{
钴(II)-三联吡啶配位聚合物催化的苄位直接氧化
}

\author{
刘建奇＼cjkstart范伟伟*＼cjkstart熊航行江京耘詹红菊 \\ (荆楚理工学院化工与药学院 湖北荆门 448000)
}

\begin{abstract}
摘要 通过苄位 $\mathrm{C}-\mathrm{H}$ 直接氧化制备了一系列芳香酮类化合物. 该反应体系采用钴(II)-三联吡啶配位聚合物作为催化 剂, 叔丁基过氧化氢(TBHP)作为氧化剂, $\mathrm{Na}_{2} \mathrm{CO}_{3}$ 作为助催化剂，水作为溶剂，以较高的收率(78\% 99\%)制得了 19 种芳 香酮类化合物. 该方法底物适用范围广，具有较高的化学选择性和官能团耐受性. 通过 2,2,6,6-四甲基哌啶-1-氧 (TEMPO)的自由基捕获实验及对反应过程中中间体的监测，提出了一种合理的自由基反应机理.

关键词 茮位直接氧化; 配位聚合物; 叔丁基过氧化氢; 酮类
\end{abstract}

\section{Benzylic Oxidation Catalyzed by Cobalt(II)-Terpyridine Coordination Polymers}

\author{
Liu, Jianqi Fan, Weiwei* Xiong, Hangxing Jiang, Jingyun $\quad$ Zhan, Hongju \\ (College of Chemical Engineering and Pharmacy, Jingchu University of Technology, Jingmen, Hubei 448000)
}

\begin{abstract}
Direct benzylic $\mathrm{C}-\mathrm{H}$ oxidation for the synthesis of aromatic ketones was developed. Employing cobalt(II)-terpyridine coordination polymers as catalyst, tert-butyl hydroperoxide (TBHP) as oxidant and $\mathrm{Na}_{2} \mathrm{CO}_{3}$ as activator, 19 aromatic ketones were prepared with good to excellent yields $(78 \% \sim 99 \%)$ in water. The reaction showed a broad range of substrates with good functional group tolerance and chemical selectivity. By control experiments, a trapping experiment using 2,2,6,6-tetramethylpiperidine-1-oxyl (TEMPO) and detection of intermediates during reaction, a reasonable radical mechanism for this type of reaction was also demonstrated
\end{abstract}

Keywords direct benzylic oxidation; coordination polymer; tert-butyl hydroperoxide; ketone

\section{Introduction}

Direct $\mathrm{C}-\mathrm{H}$ oxidation is one of the most fundamental and valuable transformations in organic synthesis. ${ }^{[1-3]}$ For decades, applying direct $\mathrm{C}-\mathrm{H}$ oxidation for the synthesis of ketones has attracted extensive research interest, as aromatic ketones are important intermediates for the synthesis of pharmaceuticals, natural products, agrochemicals, etc. For example, benzoylpyridines are key intermediates for the synthesis of many pharmaceutical products. ${ }^{[4-6]}$ For metal-catalyzed $\mathrm{C}-\mathrm{H}$ oxidation, plenty of transition-metal catalysts have been developed, such as $\mathrm{Ru},{ }^{[7]} \mathrm{Re},{ }^{[8]} \mathrm{Au},{ }^{[9]}$ $\mathrm{Cu}^{[10]} \mathrm{Co},{ }^{[11]} \mathrm{Fe},{ }^{[12-13]} \mathrm{Mn},{ }^{[14,15]} \mathrm{Ni},{ }^{[16]} \mathrm{Cr}^{[17]} \mathrm{Zn},{ }^{[18]}$ and $\mathrm{V},{ }^{[19]}$ as well as $\mathrm{Pd}-\mathrm{Cu}^{[20]}$ and $\mathrm{Mo}-\mathrm{Cu}^{[21]}$ bimetal systems. In addition, several metal-free catalytic systems have been reported, including $\mathrm{N}$-hydroxyphthalimide $/ \mathrm{O}_{2},{ }^{[22]} \mathrm{N}$-heterocyclic carbenes, ${ }^{[23]} \mathrm{I}_{2} /$ pyridine/ $/ \mathrm{BuOOH}(\mathrm{TBHP}){ }^{[24]}$ and $\mathrm{KBr}-\mathrm{Oxone},{ }^{[25,26]}$ etc. Despite significant progress, there are still many challenges to overcome for catalytic $\mathrm{C}-\mathrm{H}$ oxidation. It is a growing concern that most reaction media are environmentally unfriendly, although in most known examples the reactions were carried out under mild conditions. Towards a perspective of green chemistry, the use of benign oxidizers $\left(\mathrm{O}_{2}, \mathrm{H}_{2} \mathrm{O}_{2}\right.$, TBHP, etc. $)$ and greener solvents such as $\mathrm{H}_{2} \mathrm{O}$ and alcohols are desired. ${ }^{[7,27,28]}$

In metal catalysis, the replacement of noble metals with earth-abundant metals is a desirable aim. Employing nitrogen based ligands instead of expensive, air-sensitive and toxic phosphorus ligands is also highly anticipated. To address this, a group of cobalt and manganese coordination polymers based on readily available 4'-(4-pyridyl)$2,2^{\prime}: 6^{\prime}, 2^{\prime \prime}$-terpyridine ligands have been previously developed by the Zhang group. ${ }^{[29-33]}$ These catalysts were airand bench-stable, and exhibited excellent catalytic efficiency for carbonyl, alkene and alkyne hydroboration or

\footnotetext{
* Corresponding author. E-mail: fwwjm@foxmail.com

Received April 14, 2021; revised June 10, 2021; published online August 9, 2021.

Project supported by the Hubei Provincial Scientific and Technological Innovation Team Project (No. T2020023) and the Science and Technology Plan Project of Jingmen City (No. 2020ZDYF002).

湖北省科技创新群体项目(No. T2020023)和荆门市科技计划(No. 2020ZDYF002)资助项目.
} 
hydrofunctionalization. We were interested in exploring whether such metal coordination polymers could be utilized in oxidation catalysis. Herein, we report the facile and mild oxidation of benzylic $\mathrm{C}-\mathrm{H}$ bonds of aryl ketones with TBHP in aqueous media.

Recently, a metal-free TBHP system for benzylic oxidation has been revealed, ${ }^{[34]}$ however, excessive oxidant (12 equiv.) and high temperature were required. Although several $\mathrm{Co}(\mathrm{II})$ catalysts for benzylic $\mathrm{C}-\mathrm{H}$ oxidation with TBHP, including $\mathrm{Co}$ (acac) $2, \mathrm{Co}(\mathrm{II})$ porphyrin and $\mathrm{Co}(\mathrm{II})$ complex with 2,4,6-tris(2-pyridyl)-1,3,5-triazine, have also appeared $^{[35-37]}$ (Scheme 1), none of them could be applied in aqueous media. Our study provides an alternative $\mathrm{Co}$ (II) catalyst with high catalytic activity in aqueous media, and also proposes a new model of direct $\mathrm{C}-\mathrm{H}$ oxidation that employs one-dimensional (1-D) coordination polymer (CP) instead of conventional small molecules.

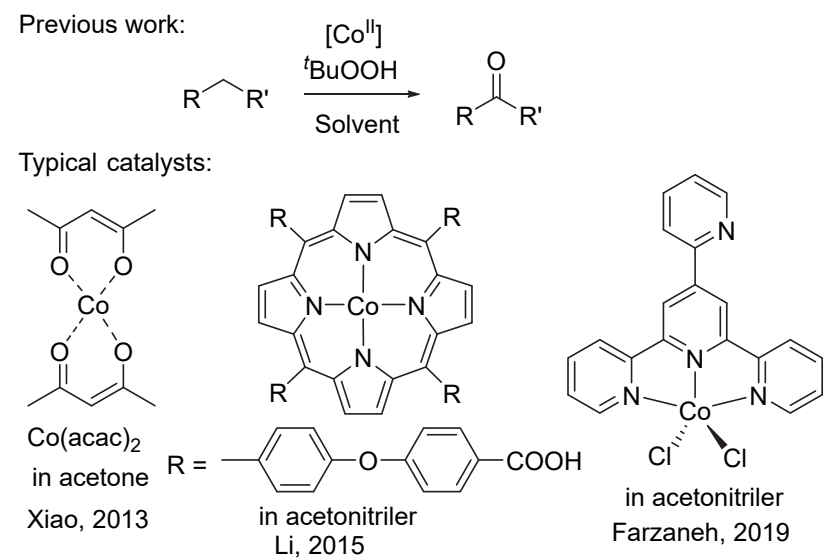

This work:

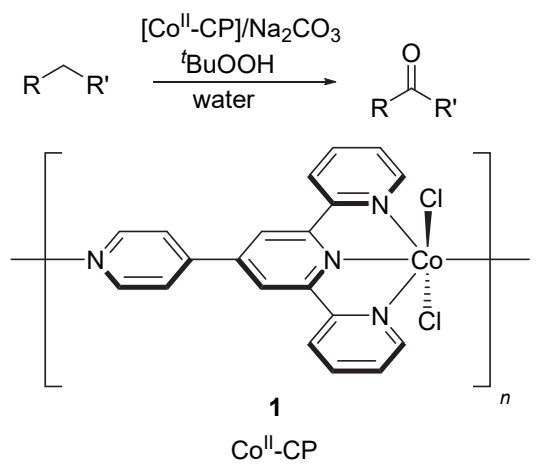

Scheme 1 Benzylic $\mathrm{C}-\mathrm{H}$ oxidation with ${ }^{t} \mathrm{BuOOH}$ catalyzed by $\mathrm{Co}$ (II) complexes

\section{Results and discussion}

To evaluate the catalytic efficiency of coordination polymer $\mathbf{1}$, the oxidation of diphenylmethane was selected as a model reaction. The influence of reaction conditions, including the catalysts, oxidants, activators and solvents was investigated (Table 1). It was pleased to observe that diphenylmethane could be efficiently oxidized by TBHP in water using 1 as a catalyst in the presence of $\mathrm{Na}_{2} \mathrm{CO}_{3}$, leading to complete conversion to benzophenone (Entry 1). Control experiments revealed that no product was observed in the absence of catalyst (Entry 2) and a much lower yield of $35 \%$ was observed with $\mathrm{CoCl}_{2} \cdot 6 \mathrm{H}_{2} \mathrm{O}$ as a catalyst (Entry 3 ), proving the role of $\mathbf{1}$ as an effective catalyst. It was further found that the choice of oxidizer is crucial to high efficiency. In the absence of TBHP or replacing it with other oxidizers $\left(\mathrm{O}_{2}, \mathrm{H}_{2} \mathrm{O}_{2},{ }^{t} \mathrm{BuOO}{ }^{t} \mathrm{Bu}\right)$, the oxidation of diphenylmethane did not occur at all (Entries $4 \sim 7$ ). The solvent effect was also found to be significant. The yields dramatically fall in other solvents such as $\mathrm{MeCN}, \mathrm{MeOH}$, dioxane or $\mathrm{EtOH}$ (Entries 8 11). Adding a base as an activator is also a key to high efficiency, which could significantly increase yield from $13 \%$ to $99 \%$ (Entries 1 and 12). But the selection of base additive was found to be less important. The reactions with $\mathrm{K}_{2} \mathrm{CO}_{3}, \mathrm{NaOH}, \mathrm{Et}_{3} \mathrm{~N}$ or ${ }^{t} \mathrm{BuOK}$ as an activator were all effective, offering excellent yields ranging from $90 \%$ to $99 \%$ (Entries $13 \sim 16$ ).

Table 1 Optimization of reaction conditions ${ }^{a}$

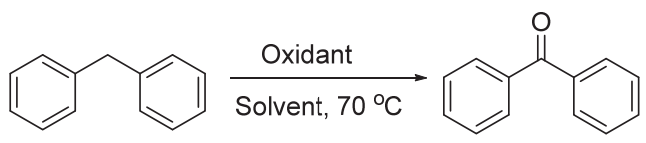

\begin{tabular}{|c|c|c|c|c|c|c|}
\hline Entry & Catalyst & Oxidant & Activator & Solvent & Time/h & Yield $^{b} / \%$ \\
\hline 1 & 1 & TBHP & $\mathrm{Na}_{2} \mathrm{CO}_{3}$ & $\mathrm{H}_{2} \mathrm{O}$ & 7 & $>99$ \\
\hline 2 & - & TBHP & $\mathrm{Na}_{2} \mathrm{CO}_{3}$ & $\mathrm{H}_{2} \mathrm{O}$ & 10 & - \\
\hline 3 & $\mathrm{CoCl}_{2} \bullet 6 \mathrm{H}_{2} \mathrm{O}$ & TBHP & $\mathrm{Na}_{2} \mathrm{CO}_{3}$ & $\mathrm{H}_{2} \mathrm{O}$ & 10 & 35 \\
\hline 4 & 1 & - & $\mathrm{Na}_{2} \mathrm{CO}_{3}$ & $\mathrm{H}_{2} \mathrm{O}$ & 10 & - \\
\hline 5 & 1 & $\mathrm{O}_{2}{ }^{c}$ & $\mathrm{Na}_{2} \mathrm{CO}_{3}$ & $\mathrm{H}_{2} \mathrm{O}$ & 10 & - \\
\hline 6 & 1 & $\mathrm{H}_{2} \mathrm{O}_{2}$ & $\mathrm{Na}_{2} \mathrm{CO}_{3}$ & $\mathrm{H}_{2} \mathrm{O}$ & 10 & - \\
\hline 7 & 1 & ${ }^{t} \mathrm{BuOO}^{t} \mathrm{Bu}$ & $\mathrm{Na}_{2} \mathrm{CO}_{3}$ & $\mathrm{H}_{2} \mathrm{O}$ & 10 & - \\
\hline 8 & 1 & TBHP & $\mathrm{Na}_{2} \mathrm{CO}_{3}$ & $\mathrm{MeCN}$ & 10 & 62 \\
\hline 9 & 1 & TBHP & $\mathrm{Na}_{2} \mathrm{CO}_{3}$ & $\mathrm{MeOH}$ & 10 & 33 \\
\hline 10 & 1 & TBHP & $\mathrm{Na}_{2} \mathrm{CO}_{3}$ & Dioxane & 10 & 6 \\
\hline 11 & 1 & TBHP & $\mathrm{Na}_{2} \mathrm{CO}_{3}$ & EtOH & 10 & 11 \\
\hline 12 & 1 & TBHP & - & $\mathrm{H}_{2} \mathrm{O}$ & 7 & 13 \\
\hline 13 & 1 & TBHP & $\mathrm{K}_{2} \mathrm{CO}_{3}$ & $\mathrm{H}_{2} \mathrm{O}$ & 7 & $>99$ \\
\hline 14 & 1 & TBHP & $\mathrm{NaOH}$ & $\mathrm{H}_{2} \mathrm{O}$ & 7 & $>99$ \\
\hline 15 & 1 & TBHP & $\mathrm{Et}_{3} \mathrm{~N}$ & $\mathrm{H}_{2} \mathrm{O}$ & 7 & $>99$ \\
\hline 16 & 1 & TBHP & ${ }^{t} \mathrm{BuOK}$ & $\mathrm{H}_{2} \mathrm{O}$ & 7 & 90 \\
\hline
\end{tabular}

Under the optimal reaction conditions, the scope of benzylic substrates was investigated, and the results are summarized in Table 2. For diphenylmethane type substrates (Table 2, 2a $\sim \mathbf{2 c}$ ), substituted benzophenones were observed with excellent yields (95\% 99\%), and functional groups such as $\mathrm{Cl}$ and $\mathrm{COOH}$ were tolerated, even though it was assumed that the introduction of $\mathrm{COOH}$ group would consume the alkali activator and thus inhibited the reaction. One possible explanation is that the substrate is insoluble in water and the tardy neutralization reaction could not prevent the activator from promoting the catalytic process. For fluorene type substrates $(\mathbf{2 d} \sim \mathbf{2 g})$, the reaction afforded oxidation products with high to excellent yields $(80 \% \sim 99 \%)$ and some electron-withdrawing groups (Br and acetyl) were tolerant. However, the intro- 
duction of stronger electron-withdrawing group such as $\mathrm{NO}_{2}$ caused an evident decline of yield (2g). 9,10-Dihydroanthracene was oxidized completely on its both benzyl sites to afford anthraquinone with a high yield (2h). 4-Benzylpyridine and xanthene were also suitable for this method, showing excellent yields $(\mathbf{2} \mathbf{i}, \mathbf{2} \mathbf{j})$. In addition, the catalytic method has been successfully applied to ethylbenzene and its derivatives, and good to excellent yields $(78 \% \sim 95 \%)$ were observed $(\mathbf{2 k} \sim \mathbf{2 s})$. Both electron-donating group $\left(\mathrm{OCH}_{3}\right)$ and electron-withdrawing groups $(\mathrm{Cl}, \mathrm{Br}, \mathrm{I})$ were tolerated, while in the case of 2-bromoethylbenzene the yield was slightly lower, possi- bly due to the steric effect (2s).

The oxidation protocol was also applied for a gram-scale synthesis. Under the same reaction condition, a $10 \mathrm{mmol}$ scale of diphenylmethane (1.68 g) was oxidized completely, resulting in the production of $\mathbf{2 a}$ in $97 \%$ isolated yield.

Finally, the possible mechanism was investigated to gain insights into the oxidative process. As the yield did not vary under the $\mathrm{N}_{2}$ atmosphere, and the substrate could not be oxidized by $\mathrm{O}_{2}$ (Table 1, Entry 5), the TBHP is the main oxidant. In a trapping experiment using 2,2,6,6-tetramethylpiperidine-1-oxyl (TEMPO), the TEMPO adduct 4 was confirmed by LC-MS (Scheme 2, a). That indicated

Table 2 Substrate scope for the benzylic oxidation ${ }^{a}$

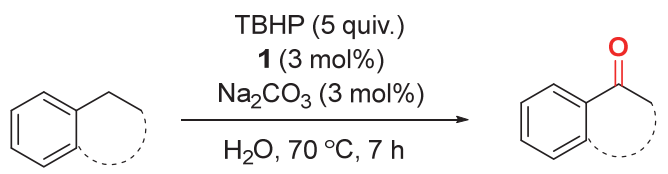<smiles>O=C(c1ccccc1)c1ccccc1</smiles>

$2 a$

$99 \%^{b}\left(99 \%^{c}\right)$<smiles>O=C1c2ccccc2-c2ccc([N+](=O)[O-])cc21</smiles>

$2 \mathrm{~g}$

$85 \%(80 \%)$<smiles>CC(=O)c1ccc(-c2ccccc2)cc1</smiles>

$93 \%(90 \%)$<smiles>O=C(c1ccccc1)c1ccc(Cl)cc1</smiles>

$\mathbf{2 b}$

$99 \%(99 \%)$<smiles>O=C1c2ccccc2C(=O)c2ccccc21</smiles>

$\mathbf{2} \mathbf{h}^{\mathrm{e}}$ $99 \%^{d}(95 \%)$<smiles>O=C(O)c1ccccc1C(=O)c1ccccc1</smiles>

2c $98 \%{ }^{d}(95 \%)$<smiles>O=C(c1ccccc1)c1ccncc1</smiles>

2i

97\% (96\%)<smiles>O=C1c2ccccc2-c2ccccc21</smiles>

2d

99\% (99\%)<smiles>O=c1c2ccccc2oc2ccccc12</smiles>

2j

99\% (97\%)<smiles>O=C1c2ccccc2-c2ccc(Br)cc21</smiles>

$2 \mathrm{e}$

95\% (93\%)<smiles>CC(=O)c1ccccc1</smiles>

$2 k$

97\% (95\%)<smiles>CCCc1ccc(C(C)=O)cc1C(=O)c1ccccc1</smiles>

99\% (98\%)<smiles>CC(=O)c1cccc2ccccc12</smiles>

2I

$96 \%^{d}(89 \%)$<smiles>COc1ccc(C(C)=O)cc1</smiles><smiles>CC(=O)c1ccc(Cl)cc1</smiles><smiles>CC(=O)c1ccc(Br)cc1</smiles><smiles>CC(=O)c1ccc(I)cc1</smiles><smiles>CC(=O)c1cccc(Br)c1</smiles><smiles>CCC(=O)c1ccccc1Br</smiles>

$2 s$

96\% (90\%)

${ }^{a}$ All reactions were performed on a 1-mmol scale in $3 \mathrm{~mL}$ solvent. ${ }^{b}$ Yield determined by GC analysis with decane as an internal standard. ${ }^{c}$ Isolated yield. ${ }^{d}$ Yield determined by ${ }^{1} \mathrm{H}$ NMR with 1,4-dioxane as an internal standard. ${ }^{e} 8$ equiv. of TBHP was added.

(a)<smiles>c1ccc(Cc2ccncc2)cc1</smiles>
optimal condition TEMPO (2.5 equiv.) $3 \mathrm{~h}$<smiles>O=C(c1ccccc1)c1ccncc1</smiles>

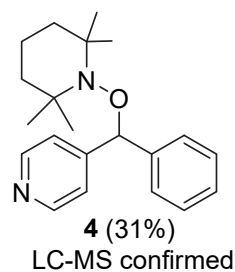

(b)<smiles>c1ccc2c(c1)Cc1ccccc1C2</smiles>
\begin{tabular}{l}
$\begin{array}{c}\text { TBHP (4 equiv.) } \\
1(3 \mathrm{~mol} \%)\end{array}$ \\
\hline $\begin{array}{l}\mathrm{Na}_{2} \mathrm{CO}_{3}(3 \mathrm{~mol} \%) \\
\mathrm{H}_{2} \mathrm{O}, 70^{\circ} \mathrm{C}, 7 \mathrm{~h}\end{array}$
\end{tabular}<smiles>c1ccc2cc3ccccc3cc2c1</smiles><smiles>O=C1c2ccccc2C(=O)c2ccccc21</smiles>
$5(44 \%)$

2h (56\%)

(c)<smiles>CC(C)OC1CCCCC1</smiles><smiles>O=C(c1ccccc1)c1ccccc1</smiles>

$2 a(42 \%)$<smiles>CCCOC(c1ccccc1)c1ccccc1</smiles>

$6(27 \%)$

Scheme 2 Trapping experiment (a), incomplete oxidation of 9,10-dihydroanthracene (b) and diphenylmethane (c) 
the abstraction of benzylic hydrogen through a radical mechanism during the reaction. This is also supported by the incomplete oxidation of 9,10-dihydroanthracene. When TBHP was inadequate (4 equiv. instead of 8 equiv.), the dehydrogenated product anthracene (5) was obtained as a byproduct (Scheme 2, b). During the oxidation of diphenylmethane, a key peroxide intermediate 6 was also observed (Scheme 2, c). It was isolated and confirmed by ${ }^{1} \mathrm{H}$ NMR and ${ }^{13} \mathrm{C}$ NMR spectroscopies.

Based on the above results, a plausible reaction mechanism was proposed as shown in Scheme 3. Firstly, Co(II) complex could react with TBHP to form a $\mathrm{Co}$ (III)-peroxy adduct, releasing ${ }^{t} \mathrm{BuOO} \bullet$ and ${ }^{t} \mathrm{BuO} \bullet$ while regenerating the Co(II) complex. ${ }^{[38-39]}$ Then the abstraction of benzylic hydrogen took place, affording radical 7 resulting from the reaction with ${ }^{t} \mathrm{BuO} \cdot{ }^{[7,21]}$ The newly generated ${ }^{t} \mathrm{BuOO} \cdot$ could also abstract benzylic hydrogens, but the rate was much less than that of $t \mathrm{BuO} \cdot{ }^{[40]}$ Therefore, it would mainly react with radical 7 to form the peroxide intermediate 6 . Finally, 6 decomposed to give the resulting ketone product. $^{[21,34]}$ It was believed that the Co(II) coordination polymers might have facilitated the decomposition of peroxide intermediate $\mathbf{6}$, as it was known that its spontaneous decomposition could only be carried out at a much higher temperature $\left(130{ }^{\circ} \mathrm{C}\right)$ with longer reaction time. ${ }^{[34]}$

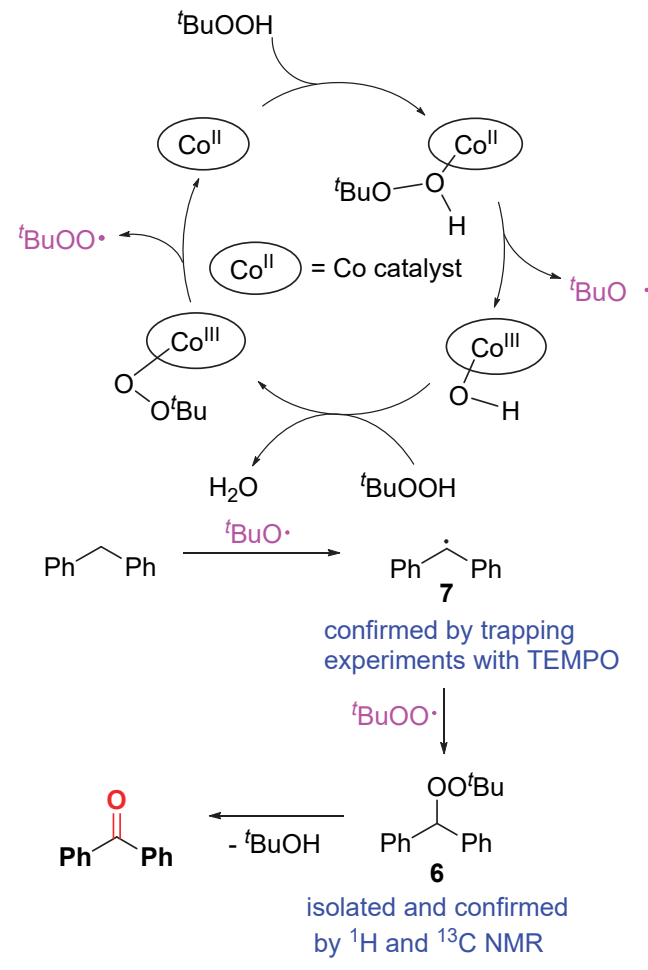

Scheme 3 Possible reaction mechanism

\section{Conclusions}

In summary, an efficient procedure for the benzylic oxidation in water, using cobalt(II)-terpyridine coordination polymer as a catalyst and TBHP as an oxidant was developed. A broad scope of substrates have been explored and good to excellent yields of the corresponding ketone products were obtained. Further studies on the structural modification of the current coordination polymer catalyst for improved performance in more challenging direct $\mathrm{C}-\mathrm{H}$ oxidation reactions are underway.

\section{Experimental section}

\subsection{Materials and methods}

All the Chemicals and solvents were purchased from commercial suppliers and used as purchased without any further purification. Unless stated otherwise, all the reactions were carried out in a $10 \mathrm{~mL}$ test tube with rubber stopper under air atmosphere. ${ }^{1} \mathrm{H} \mathrm{NMR}$ and ${ }^{13} \mathrm{C} \mathrm{NMR}$ spectra were recorded on a Bruker Avance III HD $400 \mathrm{MHz}$ spectrometer at ambient temperature with $\mathrm{CDCl}_{3}$ as solvent. For the calculation of conversions of some substrates, dioxane was added as the internal standard. Conversions of most substrates were determined by a TRACE 1300 gas chromatography equipped with a Phenomenex Zebron ZB-1701 capillary column. Mass spectrometry (LC-MS) was performed on a Thermo Scientific LCQ Fleet Ion Trap Mass Spectrometer.

\subsection{Preparation of $\mathrm{Co}$ (II) coordination polymer (cat- alyst)}

Co(II) coordination polymer was prepared according the literatures. $^{[32-33]} \quad 4^{\prime}$-(4-Pyridyl)-2,2':6',2"-terpyridine (310 $\mathrm{mg}, 1 \mathrm{mmol}$ ) was dissolved in $40 \mathrm{~mL}$ of $\mathrm{CH}_{2} \mathrm{Cl}_{2}$ and then placed in a $100 \mathrm{~mL}$ test tube. After $20 \mathrm{~mL}$ of $\mathrm{MeOH}$ was layered on the top of the solution, a solution of $\mathrm{CoCl}_{2} \bullet 6 \mathrm{H}_{2} \mathrm{O}(238 \mathrm{mg}, 1 \mathrm{mmol})$ in $\mathrm{MeOH}(40 \mathrm{~mL})$ was slowly added. The tube was sealed and stored at room temperature. Four weeks later, the crystals were collected and washed with $\mathrm{EtOH}$, and then dried in vacuo. Finally, light brown crystals were obtained ( $285 \mathrm{mg}, 52 \%$ yield) and the structure was confirmed by a X-ray single crystal diffractometer.

\subsection{General oxidation procedure}

Substrate ( $1 \mathrm{mmol})$, TBHP $(0.65 \mathrm{~g}, 70 \%$ in water), Co(II) coordination polymer (13 $\mathrm{mg}, 0.03 \mathrm{mmol}$ based on $\left.\mathrm{Co}(\mathrm{L}) \mathrm{Cl}_{2}\right), \mathrm{Na}_{2} \mathrm{CO}_{3}(3.2 \mathrm{mg}, 0.03 \mathrm{mmol})$ and water $(3 \mathrm{~mL})$ were added to a $10 \mathrm{~mL}$ test tube equipped with a stir bar. The reaction mixture was stirred at $70{ }^{\circ} \mathrm{C}$ for $7 \mathrm{~h}$. After the reaction, the reaction mixture was cooled down to room temperature and extracted 3 times with $\mathrm{CH}_{2} \mathrm{Cl}_{2}(10 \sim$ $15 \mathrm{~mL}$ ). The $\mathrm{CH}_{2} \mathrm{Cl}_{2}$ solution was diluted and analyzed by GC with decane as an internal standard. For substrates 2c, $\mathbf{2 h}$ and $\mathbf{2 l}$, the $\mathrm{CH}_{2} \mathrm{Cl}_{2}$ solution was evaporated and analyzed by ${ }^{1} \mathrm{H}$ NMR with 1,4-dioxane as an internal standard.

In a repeat experiment, the $\mathrm{CH}_{2} \mathrm{Cl}_{2}$ solution was dried with anhydrous sodium sulfate and then evaporated. The crude product was purified through silica gel column chromatography using petroleum ether and ethyl acetate as eluent.

Benzophenone (2a): White solid (181 mg, 99\% yield). m.p. $46 \sim 48{ }^{\circ} \mathrm{C}\left(\right.$ lit. $\left.{ }^{[7]} 47.5 \sim 47.9{ }^{\circ} \mathrm{C}\right) ;{ }^{1} \mathrm{H}$ NMR $(400$ 
$\left.\mathrm{MHz}, \mathrm{CDCl}_{3}\right) \delta: 7.80(\mathrm{dt}, J=8.4,1.6 \mathrm{~Hz}, 4 \mathrm{H}), 7.63 \sim 7.53$ $(\mathrm{m}, 2 \mathrm{H}), 7.52 \sim 7.43(\mathrm{~m}, 4 \mathrm{H}) ;{ }^{13} \mathrm{C} \mathrm{NMR}(101 \mathrm{MHz}$, $\left.\mathrm{CDCl}_{3}\right) \delta: 196.82,137.52,132.44,130.07,128.27$.

(4-Chlorophenyl)(phenyl)methanone (2b): White solid (216 mg, 99\% yield). m.p. $75 \sim 78{ }^{\circ} \mathrm{C}\left(\right.$ (lit. $^{[41]} 77.8 \sim$ $\left.78.4{ }^{\circ} \mathrm{C}\right) ;{ }^{1} \mathrm{H}$ NMR $\left(400 \mathrm{MHz}, \mathrm{CDCl}_{3}\right) \delta: 7.80 \sim 7.70(\mathrm{~m}$, $4 \mathrm{H}), 7.64 \sim 7.57(\mathrm{~m}, 1 \mathrm{H}), 7.52 \sim 7.42(\mathrm{~m}, 4 \mathrm{H}) ;{ }^{13} \mathrm{C} \mathrm{NMR}$ $\left(101 \mathrm{MHz}, \mathrm{CDCl}_{3}\right) \delta: 195.43,138.85,137.19,135.83$, $132.63,131.45,129.91,128.61,128.39$.

2-Benzoylbenzoic acid (2c): ${ }^{[42]}$ White solid $(215 \mathrm{mg}$, 95\% yield). m.p. $126 \sim 128{ }^{\circ} \mathrm{C}\left(123 \sim 125{ }^{\circ} \mathrm{C}\right)$; ${ }^{1} \mathrm{H}$ NMR $\left(400 \mathrm{MHz}, \mathrm{CDCl}_{3}\right) \delta: 8.07(\mathrm{~d}, J=7.8 \mathrm{~Hz}, 1 \mathrm{H}), 7.68(\mathrm{ddd}$, $J=10.3,8.7,4.2 \mathrm{~Hz}, 3 \mathrm{H}), 7.56(\mathrm{ddd}, J=10.2,8.4,4.3 \mathrm{~Hz}$, 2H), 7.40 (dd, $J=16.4,8.5 \mathrm{~Hz}, 3 \mathrm{H}) ;{ }^{13} \mathrm{C} \mathrm{NMR}(101 \mathrm{MHz}$, $\left.\mathrm{CDCl}_{3}\right) \quad \delta: 197.11,170.71,142.60,136.96,133.28$, $133.26 \sim 132.94(\mathrm{~m}), 130.92,129.58,129.45,128.48$, $127.85,127.71$.

9H-Fluoren-9-one (2d): Yellow solid (1789 mg, 99\% yield). m.p. $83 \sim 84{ }^{\circ} \mathrm{C}\left(\right.$ lit. $\left.{ }^{[43]} 79 \sim 81{ }^{\circ} \mathrm{C}\right) ;{ }^{1} \mathrm{H}$ NMR $(400$ $\left.\mathrm{MHz} \mathrm{CDCl}_{3}\right) \delta: 7.65 \sim 7.56(\mathrm{~m}, 2 \mathrm{H}), 7.48 \sim 7.39(\mathrm{~m}, 4 \mathrm{H})$, $7.30 \sim 7.20(\mathrm{~m}, 2 \mathrm{H}) ;{ }^{13} \mathrm{C} \mathrm{NMR}\left(101 \mathrm{MHz}, \mathrm{CDCl}_{3}\right) \delta$ : 193.97, 144.42, 134.72, 134.12, 129.09, 124.31, 120.34 .

2-Bromo-9H-fluoren-9-one (2e): Yellow solid (241 mg, $93 \%$ yield). m.p. $145 \sim 146{ }^{\circ} \mathrm{C}\left(\right.$ lit. $\left.^{[43]} 143 \sim 146{ }^{\circ} \mathrm{C}\right) ;{ }^{1} \mathrm{H}$ NMR (400 MHz, $\left.\mathrm{CDCl}_{3}\right) \delta: 7.76(\mathrm{~d}, J=1.8 \mathrm{~Hz}, 1 \mathrm{H}), 7.66$ $(\mathrm{dt}, J=7.3,0.8 \mathrm{~Hz}, 1 \mathrm{H}), 7.61(\mathrm{dd}, J=7.9,1.9 \mathrm{~Hz}, 1 \mathrm{H})$, $7.53 \sim 7.49(\mathrm{~m}, 2 \mathrm{H}), 7.40(\mathrm{~d}, J=7.9 \mathrm{~Hz}, 1 \mathrm{H}), 7.32(\mathrm{ddd}$, $J=7.4,5.0,3.5 \mathrm{~Hz}, 1 \mathrm{H}) ;{ }^{13} \mathrm{C} \mathrm{NMR}\left(101 \mathrm{MHz}, \mathrm{CDCl}_{3}\right) \delta$ : $192.44,143.68,143.01,137.11,135.76,135.05,133.69$, $129.44,127.58,124.63,122.92,121.74,120.45$.

2-Acetyl-9H-fluoren-9-one (2f): Yellow solid (218 mg, $98 \%$ yield). m.p. $156 \sim 158{ }^{\circ} \mathrm{C}\left(\right.$ lit. $\left.{ }^{[43]} 153 \sim 156{ }^{\circ} \mathrm{C}\right) ;{ }^{1} \mathrm{H}$ NMR (400 MHz, $\left.\mathrm{CDCl}_{3}\right) \delta: 8.29 \sim 8.05(\mathrm{~m}, 2 \mathrm{H}), 7.73(\mathrm{~d}$, $J=7.4 \mathrm{~Hz}, 1 \mathrm{H}), 7.64(\mathrm{dd}, J=11.4,4.0 \mathrm{~Hz}, 2 \mathrm{H}), 7.56(\mathrm{tt}$, $J=8.9,4.4 \mathrm{~Hz}, 1 \mathrm{H}), 7.40(\mathrm{td}, J=7.4,1.0 \mathrm{~Hz}, 1 \mathrm{H}), 2.65$ (s, $3 \mathrm{H}) ;{ }^{13} \mathrm{C} \mathrm{NMR}\left(101 \mathrm{MHz}, \mathrm{CDCl}_{3}\right) \delta: 196.64,192.79$, $148.50,143.26,137.78,135.06,134.92,134.87,134.31$, $130.25,124.68,124.19,121.29,120.46,26.78$.

2-Nitro-9H-fluoren-9-one (2g): Yellow solid $(180 \mathrm{mg}$, $80 \%$ yield). m.p. $220 \sim 222{ }^{\circ} \mathrm{C}\left(\right.$ lit. $\left.^{[44]} 222 \sim 223{ }^{\circ} \mathrm{C}\right) ;{ }^{1} \mathrm{H}$ NMR (400 MHz, $\left.\mathrm{CDCl}_{3}\right) \delta: 8.49(\mathrm{~d}, J=2.1 \mathrm{~Hz}, 1 \mathrm{H}), 8.42$ $(\mathrm{dd}, J=8.2,2.2 \mathrm{~Hz}, 1 \mathrm{H}), 7.82 \sim 7.75(\mathrm{~m}, 1 \mathrm{H}), 7.72 \sim 7.66$ $(\mathrm{m}, 2 \mathrm{H}), 7.62(\mathrm{td}, J=7.5,1.1 \mathrm{~Hz}, 1 \mathrm{H}), 7.46(\mathrm{td}, J=7.4,1.0$ $\mathrm{Hz}, 1 \mathrm{H}) ;{ }^{13} \mathrm{C} \mathrm{NMR}\left(101 \mathrm{MHz}, \mathrm{CDCl}_{3}\right) \delta: 190.97,149.74$, $148.73,142.30,135.46,135.03,134.98,131.02,129.96$, $125.12,121.80,120.72,119.58$.

Anthracene-9,10-dione (2h): Yellow-green solid (198 mg, 95\% yield). m.p. $279 \sim 281{ }^{\circ} \mathrm{C}$ (lit. ${ }^{[7]} 277.9 \sim$ $\left.278.4{ }^{\circ} \mathrm{C}\right) ;{ }^{1} \mathrm{H}$ NMR $\left(400 \mathrm{MHz}, \mathrm{CDCl}_{3}\right) \delta: 8.32(\mathrm{dt}, J=$ 7.0, $3.5 \mathrm{~Hz}, 4 \mathrm{H}), 7.84 \sim 7.78(\mathrm{~m}, 4 \mathrm{H}) ;{ }^{13} \mathrm{C}$ NMR $(101$ $\left.\mathrm{MHz}, \mathrm{CDCl}_{3}\right) \delta: 183.18,134.14,133.52,127.24$.

Phenyl(pyridin-4-yl)methanone (2i): Yellow solid (176 mg, 96\% yield). m.p. 69 $71{ }^{\circ} \mathrm{C}\left(\right.$ lit. $\left.{ }^{[4]} 68 \sim 70{ }^{\circ} \mathrm{C}\right) ;{ }^{1} \mathrm{H}$ NMR $\left(400 \mathrm{MHz}, \mathrm{CDCl}_{3}\right) \delta: 8.82(\mathrm{dd}, J=4.4,1.6 \mathrm{~Hz}, 2 \mathrm{H})$, $7.90 \sim 7.77(\mathrm{~m}, 2 \mathrm{H}), 7.72 \sim 7.62(\mathrm{~m}, 1 \mathrm{H}), 7.59(\mathrm{dd}, J=4.4$, $1.6 \mathrm{~Hz}, 2 \mathrm{H}), 7.56 \sim 7.49(\mathrm{~m}, 2 \mathrm{H}) ;{ }^{13} \mathrm{C}$ NMR $(101 \mathrm{MHz}$, $\left.\mathrm{CDCl}_{3}\right) \delta: 195.21,150.40,144.37,135.89,133.58,130.17$,
128.69, 122.91.

9H-Xanthen-9-one (2j): Beige solid (190 mg, 97\% yield). m.p. $172 \sim 174{ }^{\circ} \mathrm{C}$ (lit. $\left.{ }^{[7]} 172.9 \sim 173.4{ }^{\circ} \mathrm{C}\right) ;{ }^{1} \mathrm{H}$ NMR (400 MHz, $\left.\mathrm{CDCl}_{3}\right) \delta: 8.36(\mathrm{dd}, J=8.0,1.6 \mathrm{~Hz}, 2 \mathrm{H})$, 7.74 (ddd, $J=8.7,7.1,1.7 \mathrm{~Hz}, 2 \mathrm{H}), 7.51$ (dd, $J=8.4,0.6$ $\mathrm{Hz}, 2 \mathrm{H}), 7.39$ (ddd, $J=8.1,7.2,1.0 \mathrm{~Hz}, 2 \mathrm{H}) ;{ }^{13} \mathrm{C}$ NMR $\left(101 \mathrm{MHz}, \mathrm{CDCl}_{3}\right) \delta: 177.27,156.21,134.84,126.77$, $123.93,121.88,118.00$.

Acetophenone (2k): ${ }^{[7]}$ Colorless oil (115 mg, 95\% yield). ${ }^{1} \mathrm{H}$ NMR (400 MHz, $\left.\mathrm{CDCl}_{3}\right) \delta: 7.97$ (dt, $J=8.5,1.7$ $\mathrm{Hz}, 2 \mathrm{H}), 7.61 \sim 7.53(\mathrm{~m}, 1 \mathrm{H}), 7.52 \sim 7.42(\mathrm{~m}, 2 \mathrm{H}), 2.61(\mathrm{~s}$, $3 \mathrm{H}) ;{ }^{13} \mathrm{C} \mathrm{NMR}\left(101 \mathrm{MHz}, \mathrm{CDCl}_{3}\right) \delta: 198.25,137.11$, 133.16, 128.60, 128.34, 26.68.

1-Naphthalen-1-yl-ethanone (2l): ${ }^{[41]}$ Colorless oil (151 $\mathrm{mg}, 89 \%$ yield). ${ }^{1} \mathrm{H}$ NMR (400 $\left.\mathrm{MHz}, \mathrm{CDCl}_{3}\right) \delta: 8.83 \sim$ $8.69(\mathrm{~m}, 1 \mathrm{H}), 7.96 \sim 7.81(\mathrm{~m}, 3 \mathrm{H}), 7.58(\mathrm{ddd}, J=8.5,6.8$, $1.5 \mathrm{~Hz}, 1 \mathrm{H}), 7.52 \sim 7.41(\mathrm{~m}, 2 \mathrm{H}), 2.70(\mathrm{~s}, 3 \mathrm{H}) ;{ }^{13} \mathrm{C} \mathrm{NMR}$ $\left(101 \mathrm{MHz}, \mathrm{CDCl}_{3}\right) \delta: 201.81,135.34,133.93,133.02$, $130.09,128.70,128.38,128.02,126.40,125.97,124.30$, 29.94 .

4-Acetylbiphenyl (2m): Beige solid (176 mg, 90\% yield). m.p. $120 \sim 122{ }^{\circ} \mathrm{C}\left(\right.$ lit. $\left.^{[7]} 128.4 \sim 128.8{ }^{\circ} \mathrm{C}\right) ;{ }^{1} \mathrm{H}$ NMR $\left(400 \mathrm{MHz}, \mathrm{CDCl}_{3}\right) \delta: 8.10 \sim 7.99(\mathrm{~m}, 2 \mathrm{H}), 7.73 \sim$ $7.67(\mathrm{~m}, 2 \mathrm{H}), 7.67 \sim 7.60(\mathrm{~m}, 2 \mathrm{H}), 7.53 \sim 7.45(\mathrm{~m}, 2 \mathrm{H})$, $7.41(\mathrm{~d}, J=7.3 \mathrm{~Hz}, 1 \mathrm{H}), 2.65(\mathrm{~s}, 3 \mathrm{H}) ;{ }^{13} \mathrm{C} \mathrm{NMR}(101 \mathrm{MHz}$, $\left.\mathrm{CDCl}_{3}\right) \delta: 197.8,145.8,139.88,135.8,128.9,128.9,128.2$, $127.2,127.2,26.6$.

1-(4-Methoxyphenyl)ethanone (2n): White solid (129 mg, 86\% yield). m.p. $37 \sim 38{ }^{\circ} \mathrm{C}\left(\right.$ lit. $\left.{ }^{[7]} 38.2 \sim 38.5{ }^{\circ} \mathrm{C}\right) ;{ }^{1} \mathrm{H}$ NMR $\left(400 \mathrm{MHz}, \mathrm{CDCl}_{3}\right) \delta: 7.96 \sim 7.89(\mathrm{~m}, 2 \mathrm{H}), 6.96 \sim$ $6.88(\mathrm{~m}, 2 \mathrm{H}), 3.85(\mathrm{~s}, 3 \mathrm{H}), 2.54(\mathrm{~s}, 3 \mathrm{H}) ;{ }^{13} \mathrm{C} \mathrm{NMR}(101$ $\left.\mathrm{MHz}, \mathrm{CDCl}_{3}\right) \delta: 196.76,163.47,130.57,130.27,113.66$, $55.44,26.33$.

1-(4-Chlorophenyl)ethanone (2o): ${ }^{[21]}$ Colorless oil (136 mg, 88\% yield). ${ }^{1} \mathrm{H}$ NMR (400 $\left.\mathrm{MHz}, \mathrm{CDCl}_{3}\right) \delta: 7.94 \sim$ 7.78 (m, 2H), 7.40 (dd, $J=8.9,2.2 \mathrm{~Hz}, 2 \mathrm{H}), 2.57$ (s, 3H); ${ }^{13} \mathrm{C}$ NMR $\left(101 \mathrm{MHz}, \mathrm{CDCl}_{3}\right) \delta: 196.69,139.41,135.33$, $129.69,128.79,26.49$.

1-(4-Bromophenyl)ethenone (2p): White solid (173 mg, $87 \%$ yield). m.p. $50 \sim 52{ }^{\circ} \mathrm{C}$ (lit. $\left.{ }^{[7]} 51.4 \sim 52.2{ }^{\circ} \mathrm{C}\right) ;{ }^{1} \mathrm{H}$ NMR $\left(400 \mathrm{MHz}, \mathrm{CDCl}_{3}\right) \delta: 7.85 \sim 7.77(\mathrm{~m}, 2 \mathrm{H}), 7.62 \sim$ $7.53(\mathrm{~m}, 2 \mathrm{H}), 2.58(\mathrm{~s}, 3 \mathrm{H}) ;{ }^{13} \mathrm{C} \mathrm{NMR}\left(101 \mathrm{MHz}, \mathrm{CDCl}_{3}\right) \delta$ : $197.00,135.77,131.87,129.85,128.29,26.57$.

1-(4-Iodophenyl)ethanone (2q): Beige solid (219 mg, $89 \%$ yield). m.p. $82 \sim 84{ }^{\circ} \mathrm{C}$ (lit. $\left.{ }^{[7]} 84.4 \sim 84.8{ }^{\circ} \mathrm{C}\right) ;{ }^{1} \mathrm{H}$ NMR $\left(400 \mathrm{MHz}, \mathrm{CDCl}_{3}\right) \delta: 7.89 \sim 7.76(\mathrm{~m}, 2 \mathrm{H}), 7.73 \sim$ $7.60(\mathrm{~m}, 2 \mathrm{H}), 2.58(\mathrm{~s}, 3 \mathrm{H}) ;{ }^{13} \mathrm{C} \mathrm{NMR}\left(101 \mathrm{MHz}, \mathrm{CDCl}_{3}\right) \delta$ : 197.3, 137.9, 136.3, 129.7, 101.1, 26.4.

1-(3-Bromophenyl)ethanone (2r): ${ }^{[21]}$ Yellow oil (191 mg, 90\% yield). ${ }^{1} \mathrm{H}$ NMR (400 MHz, $\left.\mathrm{CDCl}_{3}\right) \delta: 8.07(\mathrm{t}$, $J=1.8 \mathrm{~Hz}, 1 \mathrm{H}), 7.93 \sim 7.81(\mathrm{~m}, 1 \mathrm{H}), 7.68(\mathrm{ddd}, J=8.0$, 2.0, $1.0 \mathrm{~Hz}, 1 \mathrm{H}), 7.34(\mathrm{t}, J=7.9 \mathrm{~Hz}, 1 \mathrm{H}), 2.59(\mathrm{~s}, 3 \mathrm{H}) ;{ }^{13} \mathrm{C}$ NMR $\left(101 \mathrm{MHz}, \mathrm{CDCl}_{3}\right) \delta: 196.60,138.76,135.94$, $131.33,130.21,126.87,122.94,26.63$.

1-(2-Bromophenyl)ethanone (2s): ${ }^{[21]}$ Yellow oil (167 mg, 78\% yield). ${ }^{1} \mathrm{H}$ NMR (400 MHz, $\left.\mathrm{CDCl}_{3}\right) \delta: 7.61$ (dd, $J=7.9,1.0 \mathrm{~Hz}, 1 \mathrm{H}), 7.47$ (dd, $J=7.6,1.7 \mathrm{~Hz}, 1 \mathrm{H}), 7.37$ 
(td, $J=7.5,1.1 \mathrm{~Hz}, 1 \mathrm{H}), 7.32 \sim 7.26(\mathrm{~m}, 1 \mathrm{H}), 2.63(\mathrm{~s}, 3 \mathrm{H})$;

${ }^{13} \mathrm{C}$ NMR $\left(101 \mathrm{MHz}, \mathrm{CDCl}_{3}\right) \delta: 201.42,141.46,133.86$, 131.83, 128.94, 127.47, 118.92, 30.36 .

\subsection{Gram-scale reaction}

Diphenylmethane $(1.7 \mathrm{~g})$, TBHP $(6.5 \mathrm{~g}, 70 \%$ in water $)$, $\mathrm{Co}(\mathrm{II})$ coordination polymer $(100 \mathrm{mg}), \mathrm{Na}_{2} \mathrm{CO}_{3}(30 \mathrm{mg})$ and water $(30 \mathrm{~mL})$ were added to a $100 \mathrm{~mL}$ flask equipped with a stir bar. The reaction mixture was stirred at $70{ }^{\circ} \mathrm{C}$ for $7 \mathrm{~h}$. After the reaction, the reaction mixture was cooled down to room temperature and extracted 3 times with $\mathrm{CH}_{2} \mathrm{Cl}_{2}(15 \mathrm{~mL})$. The $\mathrm{CH}_{2} \mathrm{Cl}_{2}$ solution was then evaporated. The crude product was purified through silica gel column chromatography using petroleum ether and ethyl acetate as eluent.

\subsection{Trapping experiment}

4-Benzylpyridine (169 mg, $1 \mathrm{mmol})$, TBHP (0.65 g, $70 \%$ in water), TEMPO (780 mg, $5 \mathrm{mmol}), \mathrm{Co}(\mathrm{II})$ coordination polymer $\left(13 \mathrm{mg}, 0.03 \mathrm{mmol}\right.$ based on $\left.\mathrm{Co}(\mathrm{L}) \mathrm{Cl}_{2}\right)$, $\mathrm{Na}_{2} \mathrm{CO}_{3}(3.2 \mathrm{mg}, 0.03 \mathrm{mmol})$ and water $(3 \mathrm{~mL})$ were added to a $10 \mathrm{~mL}$ test tube equipped with a stir bar. The reaction mixture was stirred at $70{ }^{\circ} \mathrm{C}$ for $7 \mathrm{~h}$. After the reaction, the reaction mixture was cooled down to room temperature and extracted 3 times with $\mathrm{CH}_{2} \mathrm{Cl}_{2}(10 \sim 15 \mathrm{~mL})$. The $\mathrm{CH}_{2} \mathrm{Cl}_{2}$ solution was diluted and analyzed by LC-MS. The products were isolated by silica gel column chromatography using petroleum ether and ethyl acetate as eluent.

Supporting Information Preparation of catalyst, ${ }^{1} \mathrm{H}$ NMR and ${ }^{13} \mathrm{C}$ NMR spectra, LC-MS spectrum. The Supporting Information is available free of charge via the Internet at http://sioc-journal.cn.

\section{References}

[1] Karimov, R. R.; Hartwig, J. F. Angew. Chem., Int. Ed. 2018, 57, 4234.

[2] Wu, J.; Zhu, J.; Li, H.; Wu, C.; Shen, R.; Yu, L. Chin. J. Org. Chem. 2019, 39, 3328 (in Chinese) (吴锦雯, 朱佳雯, 李慧, 吴春雷, 沈润溥, 余乐茂, 有机化学, 2019, 39, 3328.)

[3] Sun, Q.; Sun, W. Chin. J. Org. Chem. 2020, 40, 3686 (in Chinese). (孙强盛，孙伟，有机化学, 2020, 40, 3686.)

[4] Ren, L.; Wang, L.; Lv, Y.; Li, G.; Gao, S. Org. Lett. 2015, 17, 2078.

[5] Ren, L.; Gao, S. Chin. J. Org. Chem. 2017, 37, 1338 (in Chinese). (任兰会，高爽，有机化学, 2017, 37, 1338.)

[6] Pieber, B.; Kappe, C. O. Green Chem. 2013, 15, 320.

[7] Bo, C.; Bu, Q.; Li, X.; Ma, G.; Wei, D.; Guo, C.; Dai, B.; Liu, N. J. Org. Chem. 2020, 85, 4324.

[8] Peng, H.; Lin, A.; Zhang, Y.; Jiang, H.; Zhou, J.; Cheng, Y.; Zhu, C.; Hu, H. ACS Catal. 2012, 2, 163.

[9] Li, H.; Li, Z.; Shi, Z. Tetrahedron 2009, 65, 1856.
[10] Sterckx, H.; De Houwer, J.; Mensch, C.; Caretti, I.; Tehrani, K. A.; Herrebout, W. A.; Van Doorslaer, S.; Maes, B. U. W. Chem. Sci. 2016, 7,346

[11] Hruszkewycz, D. P.; Miles, K. C.; Thiel, O. R.; Stahl, S. S. Chem. Sci. 2017, 8, 1282.

[12] Miao, C.; Zhao, H.; Zhao, Q.; Xia, C.; Sun, W. Catal. Sci. Technol. 2016, 6, 1378 .

[13] Liu, P.; Liu, Y.; Wong, E. L.; Xiang, S.; Che, C. Chem. Sci. 2011, 2, 2187.

[14] Shen, D.; Miao, C.; Wang, S.; Xia, C.; Sun, W. Org. Lett. 2014, 16 , 1108.

[15] Talsi, E. P.; Samsonenko, D. G.; Ottenbacher, R. V.; Bryliakov, K. P ChemCatChem 2017, 9, 4580.

[16] Urgoitia, G.; Sanmartin, R.; Herrero, M. T.; Domínguez, E. Chem. Commun. 2015, 51, 4799.

[17] Yamazaki, S. Org. Lett. 1999, 1, 2129.

[18] Muthupandi, P.; Sekar, G. Tetrahedron Lett. 2011, 52, 692.

[19] Xia, J.; Cormier, K. W.; Chen, C. Chem. Sci. 2012, 3, 2240.

[20] Jeong, Y.; Moon, Y.; Hong, S. Org. Lett. 2015, 17, 3252.

[21] Li, P.; Wang, Y.; Wang, X.; Wang, Y.; Liu, Y.; Huang, K.; Hu, J.; Duan, L.; Hu, C.; Liu, J. J. Org. Chem. 2020, 85, 3101.

[22] Yang, G.; Zhang, Q.; Miao, H.; Tong, X.; Xu, J. Org. Lett. 2005, 7, 263.

[23] Alanthadka, A.; Devi, E. S.; Nagarajan, S.; Sridharan, V.; Suvitha, A.; Maheswari, C. U. Eur. J. Org. Chem. 2016, 2016, 4872.

[24] Zhang, J.; Wang, Z.; Wang, Y.; Wan, C.; Zheng, X.; Wang, Z. Green Chem. 2009, 11, 1973.

[25] Moriyama, K.; Takemura, M.; Togo, H. Org. Lett. 2012, 14, 2414.

[26] Yin, L.; Wu, J.; Xiao, J.; Cao, S. Tetrahedron Lett. 2012, 53, 4418.

[27] Sheldon, R. A. Chem. Soc. Rev. 2012, 41, 1437.

[28] Waffel, D.; Alkan, B.; Fu, Q.; Chen, Y.; Schmidt, S.; Schulz, C.; Wiggers, H.; Muhler, M.; Peng, B. ChemPlusChem 2019, 84, 1155.

[29] Zhang, G.; Zeng, H.; Li, S.; Johnson, J.; Mo, Z.; Neary, M. C.; Zheng, S. Dalton Trans. 2020, 49, 2610.

[30] Zhang, G.; Li, S.; Wu, J.; Zeng, H.; Mo, Z.; Davis, K.; Zheng, S. Org. Chem. Front. 2019, 6, 3228.

[31] Fan, W.; Li, L.; Zhang, G. J. Org. Chem. 2019, 84, 5987.

[32] Zhang, G.; Wu, J.; Li, S.; Cass, S.; Zheng, S. Org. Lett. 2018, 20, 7893.

[33] Wu, J.; Zeng, H.; Cheng, J.; Zheng, S.; Golen, J. A.; Manke, D. R.; Zhang, G. J. Org. Chem. 2018, 83, 9442.

[34] Tan, J.; Zheng, T.; Yu, Y.; Xu, K. RSC Adv. 2017, 7, 15176.

[35] Zhang, Z.; Li, J.; Yao, Y.; Sun, S. Cryst. Growth Des. 2015, 15, 5028.

[36] Azarkamanzad, Z.; Farzaneh, F.; Maghami, M.; Simpson, J. New J. Chem. 2019, 43, 12020.

[37] Han, X.; Zhou, Z.; Wan, C.; Xiao, Y.; Qin, Z. Synthesis 2013, 45, 615.

[38] Zhang, J.; Biradar, A. V.; Pramanik, S.; Emge, T. J.; Asefa, T.; Li, J. Chem. Commun. 2012, 48, 6541.

[39] Asgharpour, Z.; Farzaneh, F.; Abbasi, A. RSC Adv. 2016, 6, 95729.

[40] Bryant, J. R.; Mayer, J. M. J. Am. Chem. Soc. 2003, 125, 10351.

[41] Wang, T.; Jing, X.; Chen, C.; Yu, L. J. Org. Chem. 2017, 82, 9342.

[42] Cheng, K.; Zhao, B.; Qi, C. RSC Adv. 2014, 4, 48698.

[43] Li, L.; Liu, Z.; Tang, S.; Li, J.; Ren, X.; Yang, G.; Li, H.; Yuan, B. Catal. Commun. 2019, 127, 34.

[44] Zhang, X.; Ji, X.; Su, R.; Weeks, B. L.; Zhang, Z.; Deng, S. ChemPlusChem 2013, 78, 703. 